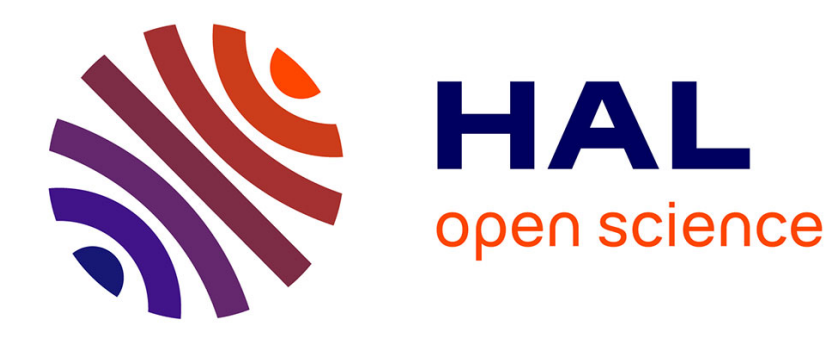

\title{
Computational polarization imaging from a single speckle image
}

Julien Fade, Muriel Roche, Mehdi Alouini

\section{To cite this version:}

Julien Fade, Muriel Roche, Mehdi Alouini. Computational polarization imaging from a single speckle image. Optics Letters, 2012, 37 (3), pp.386-388. 10.1364/OL.37.000386 . hal-00667320

\section{HAL Id: hal-00667320 \\ https://hal.science/hal-00667320}

Submitted on 19 Dec 2014

HAL is a multi-disciplinary open access archive for the deposit and dissemination of scientific research documents, whether they are published or not. The documents may come from teaching and research institutions in France or abroad, or from public or private research centers.
L'archive ouverte pluridisciplinaire HAL, est destinée au dépôt et à la diffusion de documents scientifiques de niveau recherche, publiés ou non, émanant des établissements d'enseignement et de recherche français ou étrangers, des laboratoires publics ou privés. 


\title{
Computational polarization imaging from a single speckle image
}

\author{
Julien Fade ${ }^{1, *}$, Muriel Roche ${ }^{2}$ and Mehdi Alouini ${ }^{1,3}$ \\ ${ }^{1}$ Institut de Physique de Rennes, Université de Rennes 1, CNRS, Campus de Beaulieu, 35042 \\ Rennes, France \\ ${ }^{2}$ Institut Fresnel, Aix-Marseille Université, Ecole Centrale Marseille, CNRS, Campus \\ Saint-Jérôme, 13013 Marseille, France \\ 3 Thales Research and Technology, RD 128, 91767 Palaiseau Cedex, France \\ * Corresponding author : julien.fade@univ-rennes1.fr
}

We experimentally demonstrate that imaging of the degree of polarization (DOP) can be achieved from a single intensity image acquired under coherent illumination. This computational technique is based on the analysis of the speckle characteristics in a statistically homogeneous neighborhood of the pixel. Over a variety of samples obtained experimentally, we show that a simple calibration step allows this method to quickly and simply provide correct estimated values of the DOP, with precision in agreement with theoretical predictions. It is shown that unlike linear polarimetric contrast imaging, this method remains valid on birefringent samples. (C) 2014 Optical Society of America

OCIS codes: 120.5410, 030.4280, 120.6150, 030.6600.

Polarimetric imaging is a promising and widely investigated technique in many different fields of application such as military, medical, or robotic domains $[1,2]$. In particular, imaging the degree of polarization (DOP) of the light backscattered by a scene can reveal contrasts that do not appear 


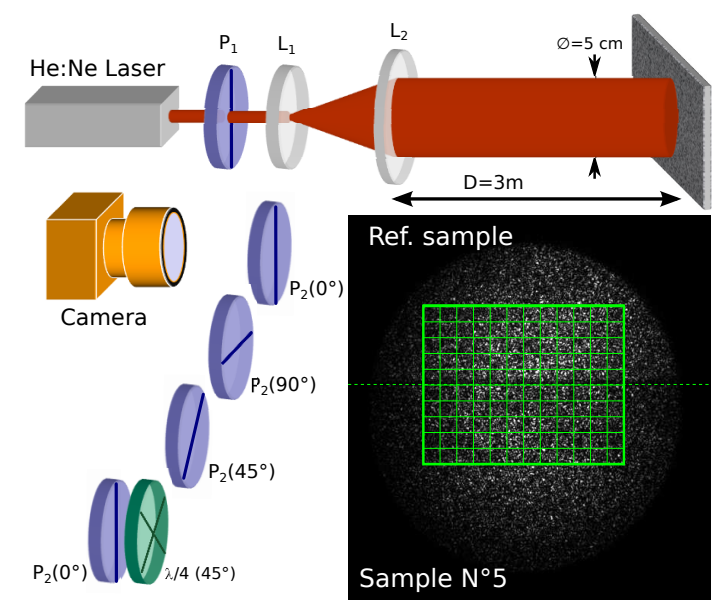

(a)

(b)

Fig. 1. (a) Experimental setup; (b) Example of an intensity image of the reference sample and sample 5 (green paint).

in standard intensity images [2]. Four polarimetric measurements are actually needed to determine the polarization state of light, but simplified imaging modalities have been proposed to reduce both costs and acquisition times, such as the Orthogonal States Contrast (OSC) imaging, requiring only two measurements [2]. Recently, theoretical studies [3-7] have shown that an alternative DOP imaging technique, using a single image acquisition under coherent active illumination and without polarizing element, could lead to estimation performances compatible with potential applications. This computational approach is based on the analysis of the speckle statistics in the image, which contain information on the DOP of the reflected light [8]. In addition to its simplicity, this technique is applicable at any wavelength and is not affected with image registration issues which are in general detrimental to the quality of polarimetric imaging devices. In this Letter, we experimentally demonstrate the applicability of such a technique over a variety of samples, and we analyze its precision in comparison to theoretical characterizations [5] and to standard methods.

The experimental setup used is sketched in Fig. 1.a. The sample is illuminated with a $5 \mathrm{~cm}$ diameter spot from a collimated $633 \mathrm{~nm}$ He:Ne laser beam of $15 \mathrm{~mW}$ power. A polarizer $\left(P_{1}\right)$ 
ensures a vertical direction of the illumination polarization. Imaging of the sample is operated with a 12-bits Basler A312f CCD camera $(782 \times 582$ pixels $)$ and a $50 \mathrm{~mm}$ objective, whose line of sight is almost aligned with the illumination beam axis (to avoid undesirable specular reflections). The camera has been selected for its good noise properties under low illumination, when the objective aperture is shrunk to obtain at least a speckle grain per pixel in average. Four Stokes images can be recorded with appropriate configurations of a polarization analyzer $\left(P_{2}\right)$ and a quarter-wave plate (see Fig. 1.a). The image of the DOP in each pixel of the scene can then be classically obtained from these 4 Stokes measurements [9]. In addition, the OSC image can also be derived from $O S C=\left(I_{/ /}-I_{\perp}\right) /\left(I_{/ /}+I_{\perp}\right)$, where $I_{/ /}$and $I_{\perp}$ denote the first two Stokes images acquired. OSC is often used in polarimetric imaging applications since it is equal to the DOP over a majority of materials (purely depolarizing materials) [2].

In addition to these 4 Stokes measurements, a fifth image denoted $I_{T}$ is recorded without any polarimetric component in front of the camera objective. As detailed in [3-7], the square DOP, denoted $\mathcal{P}^{2}$, can be retrieved very simply from this bare speckle intensity image, by computing the following estimator: $\widehat{\mathcal{P}^{2}}=2\left[\left\langle I_{T}^{2}\right\rangle_{\chi} /\left\langle I_{T}\right\rangle_{\chi}^{2}-1\right] / \kappa-1$, where $\langle\cdot\rangle_{\chi}$ denotes statistical averaging over a homogeneous neighborhood $\chi$ of the considered pixel. Previous theoretical work [5] has shown that statistically homogeneous neighborhoods of typically a few hundred pixels are needed for such a method to provide a satisfactory estimation precision (about 10\%). In the previous equation, the parameter $\kappa$ is directly linked to the speckle order $L=1 / \kappa$, which characterizes the average number of speckle grains contained in one pixel of the imaging system [5]. This computational method must therefore be calibrated to characterize the value of the parameter $\kappa$, which will be detailed in the following.

In the first experiment reported here, we have considered 8 different polarimetric scenes (see Fig. 1.b), comprising an identical reference material (beige painted cardboard slab) in the upper part 
Table 1. Description of the samples. Bracketted figures correspond to the value of $\mathcal{P}^{2}$ estimated from Stokes measurements.

\begin{tabular}{|ll|ll|}
\hline \multicolumn{2}{|l|}{ Reference: Beige cardboard (0.61) } \\
\hline 1: & Black paper (0.72) & 2: & White paper (0.04) \\
3: & Cream plastic (0.20) & 4: & Gray paint (0.60) \\
5: & Green paint (0.98) & 6: & Aluminium (0.98) \\
7: & Sandblasted Alum. (0.70) & 8: & Black plastic (0.91) \\
\hline
\end{tabular}

of the image, and 8 different test materials described in Table 1 in the lower part of the image. To avoid illumination inhomogeneities, regions of interest (ROIs) of $240 \times 100$ pixels are selected in the upper and lower parts of the images. These regions are then further partitioned in 60 sub-regions of 400 pixels each (see Fig. 1.b). Indeed, performing the estimation on such regions of limited size appears more realistic (homogeneous regions $>10^{4}$ pixels are unlikely to occur in real conditions), and smaller regions ensure good illumination uniformity. Lastly, it allows a statistical analysis of the estimation precision to be carried out from the 60 realizations.

As mentioned previously, a precise characterization of the parameter $\kappa$ is needed before the estimator $\widehat{\mathcal{P}^{2}}$ can be applied on a single intensity image. For a fixed wavelength and experiment geometry, $\kappa$ is inversely proportional to the objective aperture and does not depend on the sample considered. Computing the ratio of the variance to the square mean intensity of a fully polarized speckle pattern is a simple way to determine this parameter experimentally [8]. We thus calibrated the estimator $\widehat{\mathcal{P}^{2}}$ on the reference sample by computing $\kappa$ on the 4 Stokes images acquired (which are fully polarized). Very similar results were obtained on each Stokes image and along the 8 experiments, finally leading to the following estimated value $\hat{\kappa}=0.808 \pm 0.003$, which corresponds to a speckle order of $L=1.24$.

Once characterized the parameter $\kappa$, the estimator $\widehat{\mathcal{P}^{2}}$ can be applied on the image $I_{T}$ acquired 

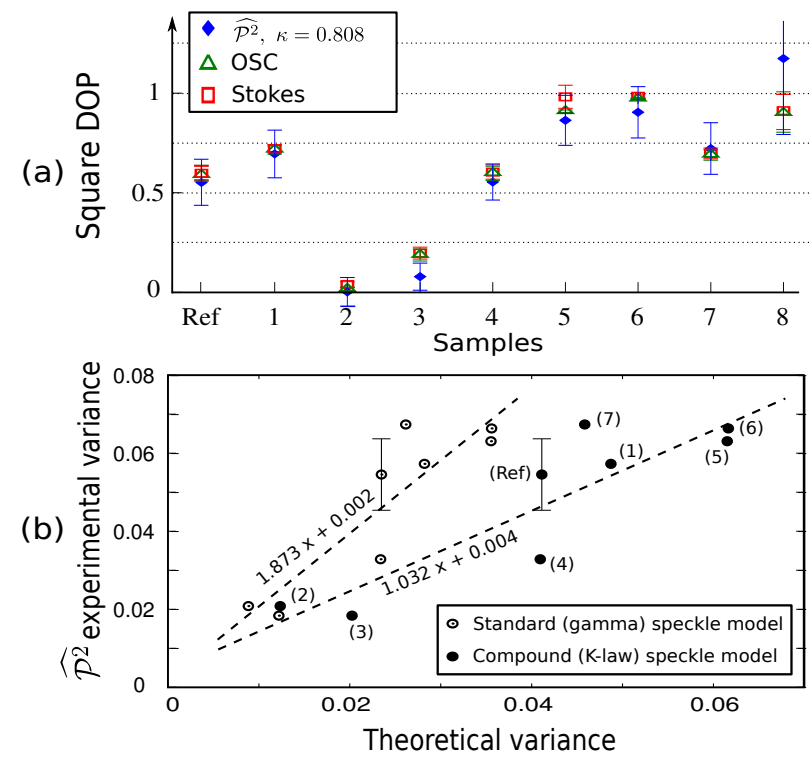

Fig. 2. (a) Values of the square DOP estimated on homogeneous regions of 400 pixels on the 9 samples. (b) Comparison between experimental variance of $\widehat{\mathcal{P}^{2}}$ and theoretical predictions for distinct speckle models. Sample 8 leading to an erroneous estimated value of $\mathcal{P}$ is not represented in this figure. The error-bar on experimental variance is estimated from the 8 experiments conducted on the reference sample.

without polarimetric component. Estimation results are plotted with blue diamond symbols in Fig. 2.a for the reference sample and for the 8 test samples. It can be immediately checked that except for test sample 8, the estimator $\widehat{\mathcal{P}^{2}}$ provides a correct value of the square DOP, in agreement (at least within the estimated error-bar) with the results obtained with standard Stokes measurements (red squares) or OSC (green triangles). This experimentally demonstrates the applicability of the computational technique addressed here. Furthermore, this result shows that the value of $\kappa$ estimated on the reference sample remains valid on other materials. Unfortunately, this might not be always the case, for instance with samples showing a non uniform reflectivity distribution or with textured materials. We suspect such a situation to occur with sample 8 for which $\widehat{\mathcal{P}^{2}}$ leads to irrelevant values $\left(\widehat{\mathcal{P}^{2}}=1.2\right)$. It has been checked that the parameter $\kappa$ was equal to 0.95 on this black plastic slab, which can be interpreted as follows: since the sample is very dark, light mainly 
undergoes surface backscattering (and hence very low depolarization [10]) on the smooth, glossy plastic surface, making it difficult to avoid detrimental contribution from specular backscattering.

As stated previously, the precision of the single-image computational estimation method can be evaluated from the 60 homogeneous regions of 400 pixels on which the estimation was operated. In Fig. 2.a, one can see that a larger standard-deviation is of course the price to pay for a simplification of the imaging system. It must however be noticed that, even on samples of limited size $(20 \times 20$ pixels), the computational estimation method allows materials of distinct polarimetric properties to be discriminated, with a $10 \%$ precision on $\mathcal{P}^{2}$. Previous theoretical results can be used to assess the variance of $\widehat{\mathcal{P}^{2}}$ for various speckle models [5]. This can be checked in Fig. 2.b, where the experimental variance of $\widehat{\mathcal{P}^{2}}$ is compared with theoretical predictions obtained when a so-called standard (resp. compound) speckle model is considered, i.e., when the intensity distribution for $\mathcal{P}=1$ is assumed to follow a Gamma distribution (resp. a $\mathcal{K}$-distribution) [8], with an appropriate shape parameter equal to $\mathrm{L}=1 / \kappa$. As can be seen from the linear fit parameters, a fair agreement between experiments and theory can be observed, especially with a compound speckle model, which seems to be more relevant in the conditions of our experiment.

Table 2. Estimated values of the square DOP for metal and cardboard samples for different speckle orders. Bracketted number is the estimation standard-deviation.

\begin{tabular}{ccccc} 
Sample & $\mathrm{f} /$. & $\kappa$ & $\widehat{\mathcal{P}_{O S C}^{2}}$ & $\widehat{\mathcal{P}^{2}}$ \\
\hline Metal & $\mathrm{f} / 16$ & $0.7[0.1]$ & $0.94[0.01]$ & $0.9[0.3]$ \\
& $\mathrm{f} / 4$ & $0.12[0.02]$ & $0.96[0.01]$ & $0.8[0.3]$ \\
\hline Card- & $\mathrm{f} / 16$ & $0.66[0.07]$ & $0.05[0.02]$ & $0.04[0.1]$ \\
board & $\mathrm{f} / 4$ & $0.14[0.02]$ & $0.06[0.01]$ & $-0.03[0.1]$ \\
\hline
\end{tabular}

To check the validity of the method when the speckle order varies, a second experiment has been conducted with a green $532 \mathrm{~nm}$ frequency-doubled $\mathrm{Nd}: \mathrm{YVO}_{4}$ laser of $10 \mathrm{~mW}$ power. Two samples 
have been imaged (a metal slab and a cardboard sheet) for two distinct objective apertures (f/16 and $\mathrm{f} / 4$ ), leading to different parameters $\kappa$ (see Table 2 ). Both measurements of $\kappa$ and $\mathcal{P}^{2}$ have been carried out on 100 homogeneous regions of $10 \times 10$ pixels. In this experiment, the calibration procedure has been performed on the cardboard reference sample. The results are summarized in Table 2. It can be checked that the single-image computational technique remains valid for different values of the parameter $\kappa$, provided it has been carefully characterized.

A last experiment is reported in this Letter to check the applicability of the single-image computational technique on non purely depolarizing materials (e.g., birefringent samples). The experimental configuration is similar to the one described in the previous paragraph, but we added a tunable Babinet-Soleil (BS) compensator within the path of the backscattered light, with slow and fast axes oriented at a $45^{\circ}$ angle from the illumination polarization direction. With the metallic sample, we were thus able to obtain an almost fully-polarized backscattered speckle field $\left(\mathcal{P}^{2}=0.93\right.$ from Stokes measurements), with a polarization state varying between a vertical linear polarization and a circular polarization, when the BS compensator retardation angle $\varphi$ was varied between 0 and $\pi / 2$. In Fig. 3, we plotted the estimation results obtained with the computational method (blue diamonds) and with the OSC (green triangles). It can first be checked on this figure that the OSC is unable to correctly evaluate the DOP in the presence of birefringent samples while estimator $\widehat{\mathcal{P}^{2}}$ remains valid for any polarization state. It can be also noted that the estimation precision does not depend on the polarization state ellipticity, in agreement with theoretical predictions plotted with a dashed error-bar for a compound speckle model.

In this Letter, a computational technique involving a single image acquisition has been tested successfully on a variety of non-textured materials to achieve DOP estimation, with precision in agreement with theory [5]. The applicability of such a technique at different wavelengths, on birefringent materials, and for distinct speckle orders has also been demonstrated. As a perspective 


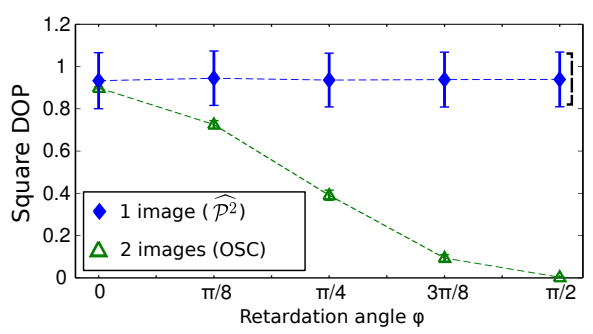

Fig. 3. Square DOP estimated from 2 images (OSC) and 1 image (estimator $\widehat{\mathcal{P}^{2}}$ ), as a function of the retardation $\varphi$ imposed by the BS compensator.

to this work, other experiments should be conducted to validate previous theoretical predictions which showed that this computational technique should be quite robust to photon noise [6] and detector noise [7], thus enabling low-flux real-time polarimetric imaging.

The authors thank Pr. Ph. Réfrégier for fruitful discussions.

\section{References}

1. J. S. Tyo, D. L. Goldstein, D. B. Chenault, and J. A. Shaw, Appl. Opt. 45, 5453 (2006).

2. S. Breugnot and P. Clémenceau, Proc. SPIE 3707, 449, (1999).

3. T. J. Schulz, Proc. SPIE 5888, 58880N (2005).

4. P. Réfrégier, J. Fade, and M. Roche, Opt. Lett. 32, 739-741 (2007).

5. J. Fade, M. Roche, and P. Réfrégier, J. Opt. Soc. Am. A 25, 483 (2008).

6. J. Fade, P. Réfrégier, and M. Roche, J. Opt. A: Pure and Appl. Opt. 10, 115301 (2008).

7. W. Wang and T. J. Schulz, J. Opt. Soc. Am. A 27, 1274 (2010).

8. J. W. Goodman, Speckle phenomena: Theory and Applications, chap. First order statistical properties of optical speckle, (Roberts \& Company Publishers, 2007).

9. E. Collett, Polarized light: fundamentals and applications (Dekker, 1993).

10. M. Alouini, F. Goudail, N. Roux, L. Le Hors, P. Hartemann, S. Breugnot, and D. Dolfi, EPJ Appl. Phys. 42, 129 (2008). 


\section{Informational Fourth Page}

\section{References}

1. J. S. Tyo, D. L. Goldstein, D. B. Chenault, and J. A. Shaw, "Review of passive imaging polarimetry for remote sensing applications," Appl. Opt. 45, 5453-5469 (2006).

2. S. Breugnot and P. Clémenceau, "Modeling and performances of a polarization active imager at lambda=806 nm," in "Laser Radar technology and applications IV," , vol. 3707, G. W. K. C. Werner, ed. (Proc. SPIE, 1999), vol. 3707, pp. 449-460.

3. T. J. Schulz, "Performance bounds for the estimation of the degree of polarization from active laser illumination," in "Polarization science and remote sensing II," (Proc SPIE vol. 5888, 2005), p. $58880 \mathrm{~N}$.

4. P. Réfrégier, J. Fade, and M. Roche, "Estimation precision of the degree of polarization from a single speckle intensity image," Opt. Lett. 32, 739-741 (2007).

5. J. Fade, M. Roche, and P. Réfrégier, "Precision of moment based estimation of the degree of polarization in coherent imagery without polarization device," Journal of the Optical Society of America A 25, 483-492 (2008).

6. J. Fade, P. Réfrégier, and M. Roche, "Estimation of the degree of polarization from a single speckle intensity image with photon noise," Journal of Optics A: Pure and Applied Optics 10, 115301 (2008).

7. W. Wang and T. J. Schulz, "Performance bounds for the estimation of the degree of polarization from various sensing modalities," J. Opt. Soc. Am. A 27, 1274-1287 (2010).

8. J. W. Goodman, Speckle phenomena: Theory and Applications (Roberts \& Company Publishers, Greenwood village, 2007), chap. First order statistical properties of optical speckle.

9. E. Collett, Polarized light: fundamentals and applications (Dekker, New-York, 1993). 
10. M. Alouini, F. Goudail, N. Roux, L. Le Hors, P. Hartemann, S. Breugnot, and D. Dolfi, “Active spectro-polarimetric imaging: signature modeling, imaging demonstrator and target detection," The European Physical Journal - Applied Physics 42, 129-139 (2008). 